\title{
Entre interrogation et exclamation
}

Destruction de l'utopie de la recherche identitaire panérienne

\section{Claire Laguian}

\section{OpenEdition}

Journals

Édition électronique

URL : http://journals.openedition.org/agedor/662

DOI : 10.4000 /agedor.662

ISSN : 2104-3353

Éditeur

Laboratoire LISAA

Référence électronique

Claire Laguian, « Entre interrogation et exclamation », L'Âge d'or [En ligne], 7 | 2014, mis en ligne le 01

mars 2014, consulté le 01 mai 2019. URL : http://journals.openedition.org/agedor/662 ; DOI :

10.4000/agedor.662

L'Âge d'or. Images dans le monde ibérique et ibéricoaméricain 


\title{
Entre interrogation et exclamation : destruction de l'utopie de la recherche identitaire panérienne
}

\begin{abstract}
Résumé : Toute quête identitaire passe par un processus d'interrogation sur le moi, et la voix poétique panérienne ne déroge pas à la règle, puisqu'elle utilise très fréquemment les questions dans ses poèmes. Néanmoins, par divers stratagèmes linguistiques, elle transgresse totalement l'obligation de réponse qui conditionne normalement l'existence de l'interrogation. L'exclamation, du fait de son caractère d'affirmation hyperbolique, semble supplanter une interrogation en échec total, surtout à partir des années 2000. Mais, la voix poétique chez Leopoldo María Panero essuie encore un non-aboutissement puisque le sémantisme de l'exclamation implique toujours une facette négative. Ce qui détruit finalement tout espoir d'une quelconque voie identitaire, capable d'être définie ou trouvée, c'est la prépondérance des interjections syllabiques, «ah» et «oh», qui s'apparentent au cri et installent paradoxalement un silence signifiant dans des poèmes qui finissent peut-être par accepter le caractère vain, utopique, de toute recherche identitaire.
\end{abstract}

Mots-clés: Leopoldo María Panero - Quête identitaire - Interrogation - Exclamation Interjection - Cri - Silence

Resumen: Cualquier búsqueda de identidad pasa por un proceso de interrogación sobre el yo, y la vOz poética paneriana no contraviene a esta regla, puesto que utiliza muy a menudo las preguntas en sus poemas. No obstante, mediante varias estratagemas lingüísticas, transgrede totalmente la obligación de tener una respuesta que condiciona normalmente la existencia de la interrogación. La exclamación, por su carácter de afirmación hiperbólica, parece sustituirse a una interrogación fallida, sobre todo a partir de los años 2000. Pero, la voz poética en Leopoldo María Panero sufre otro fracaso, ya que el campo semántico de la exclamación siempre implica una faceta negativa. Lo que finalmente destruye cualquier esperanza de vía identitaria, capaz de ser definida o encontrada, es la preponderancia de las interjecciones silábicas, "ah" y "oh", que se aparentan al grito e instalan paradójicamente un silencio significante en poemas que acaso terminen por aceptar el carácter vano, utópico, de cualquier búsqueda identitaria.

Palabras clave: Leopoldo María Panero - Búsqueda de identidad - Interrogativas - Exclamativas - Interjección - Grito - Silencio

Quién sabe quién soy yo Leopoldo María Panero.

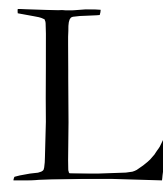

a recherche du moi poétique dans l'œuvre de Leopoldo María Panero est une problématique centrale, comme le signale le critique Túa Blesa en introduction à l'œuvre de ce poète : «la identidad del individuo puesta en crisis, alterada o directamente perdida, lo que constituye uno de los temas 
más persistentes de este conjunto poético ${ }^{1}$. Nous tenterons d'aborder ici cette errance identitaire selon une perspective strictement linguistique. Pour ce faire, il nous semble que le point de départ pourrait se situer autour de l'acte interrogatif, en tant qu'acte de parole élémentaire du questionnement identitaire. En effet, l'interrogation est le fondement de la relation à notre propre existence, comme le souligne le philosophe Francis Jacques: «Notre rapport au monde passe par un processus d'interrogation $»^{2}$, et n'est-ce pas d'ailleurs par la résolution de cet acte, exprimant intrinsèquement le doute et l'ignorance, par le sésame offert par la réponse attendue, qu'un sujet est censé avoir accès à sa vérité identitaire ? Depuis les années 70, Panero met en place dans sa poésie un système langagier très particulier, centré sur la recherche identitaire et qui nous signale par ce biais son éternelle volonté de ruiner la langue. Ainsi, nous essaierons de préciser les modalités de cette déconstruction linguistique au sein du processus de quête du moi panérien, et de voir si elles aboutissent, ou non, à une quelconque reconstruction efficace.

Tout d'abord, nous nous pencherons sur les occurrences interrogatives disséminées au long de l'œuvre de Leopoldo María Panero, en signalant en quoi elles sont toutes transgressives. Nous constaterons par la suite que la question va être éludée et vite remplacée par l'acte exclamatif qui semble être plus propice, de par sa nature linguistique, à exprimer l'identité du moi du poète. Enfin, nous verrons comment le cri et son contrepied, le silence, envahissent définitivement les vers panériens.

\section{La non-saturation des interrogations ou l'échec de la quête identitaire}

Dans les recueils panériens, nous observons une fréquence très élevée d'apparition des actes interrogatifs qui leur confèrent d'ailleurs une prosodie et une intensité marquantes : il est essentiel de signaler de prime abord que ces questions se caractérisent toutes par une absence de réponse effective, ou par une réponse inadéquate sur un plan sémantique. De la sorte, la recherche de la voix poématique ${ }^{3}$ échoue, ou commet un " accroc ${ }^{4}$ selon la terminologie d'Austin, puisque normalement la question est un véritable 《diktat $»^{5}$ qui a « le pouvoir (exorbitant) d'obliger le destinataire à continuer le discours $»^{6}$, à fournir une réponse effective et cohérente. Nous essaierons donc de relever par la suite les différentes modalités de cette destruction de la fonction élémentaire de la question. Tout d'abord, les seules réponses qui apparaissent sont soit inadéquates du fait de leur incongruité: "¿Qué es la noche? / ¿Qué es el búho? ¡Si un perro ladrara! »", soit inintelligibles de par leur caractère inarticulé : «Preguntadle a un perro: ¿qué es la locura? / y ladrará tres veces $»^{8}$. Lorsque la voix poématique s'adresse à un interlocuteur présent explicitement dans le poème, elle semble condamnée à la solitude et au vide puisqu'aucune réponse n'est apportée dans ce dialogue. En effet, ces interlocuteurs sont bien incapables, de par leur statut animal, leur mort ou leur fictionnalité littéraire, de fournir la moindre

\footnotetext{
1 BLESA, Túa, «La destruction fut ma Béatrice, I », in PANERO, Leopoldo María, Poesía Completa (19702000), Madrid, Visor Libros, 2010, p. 15.

${ }^{2}$ FRANCIS, Jacques, L'espace logique de l'interlocution, Paris, PUF, 1985, p. 322.

${ }^{3}$ Concept de « voix poématique » cher à Marie-Claire Zimmermann.

${ }_{4}^{4}$ AUSTIN, John Langshaw, Quand dire, c'est faire, Paris, Éditions du Seuil, 1970, p. 30.

${ }^{5}$ KERBRAT-ORECCHIONI, Catherine, La Question, Lyon, Presses Universitaires de Lyon, 1991, p. 28.

${ }^{6}$ DUCROT, Oswald, L'argumentation dans la langue, Bruxelles, Ed. Mardaga, 1983, p. 99.

7 PANERO, Leopoldo María, Poesía Completa (1970-2000), Madrid, Visor Libros, 2010, p. 295.

${ }^{8}$ Ibid, p. 459.
} 
réponse: "Y le pregunté a una hormiga, dije ¿Quién soy yo? y nadie respondió »", "Filóstrato, si muero ¿beberás de mis venas? »" "CAPITÁN MARVEL, ¿DÓNDE ESTÁS? " ${ }^{11}$. Dans cette dernière occurrence, le silence est d'autant plus envahissant que cette interrogation non-saturée, non-résolue, se situe à la fin du poème et laisse donc la voix poétique et le lecteur dans une expectative à jamais frustrée.

À propos de la question identitaire envahissante, "¿quién soy yo? », présente dans le soliloque du poète, nous signalerons qu'il pourrait s'agir d'une question écho, d'une question de reprise, suite à celle d'un éventuel interlocuteur qui aurait pu exprimer au moi panérien : «¿quién eres tú? ». À cette interrogation identitaire correspondent des pseudoréponses plongeant la voix poématique dans le silence : «En los atardeceres lluviosos me pregunto "¿Quién soy yo?" y la nada responde a mi llanto »" Un autre cas serait que le poème laisse cette question en suspens par la non-saturation, en l'absence de réponse, "¿QQuién soy? - grita el enano » ${ }^{13}$, et ce, même quand la question offre une possibilité de réponse en son sein. Voyons ici ces différentes suggestions de réponse intégrées à l'interrogation : «¿Quién soy yo sino un perro atado a la nada? " ${ }^{14}$ ou alors "Quién soy yo, sino una mueca ante el espanto $»^{15}$ : nous remarquons que cette proposition de réponse est très restrictive comme le prouve l'emploi de «sino ", et de plus, elle nie totalement le statut humain du locuteur ( perro», « mueca ») et remet donc en question le bien-fondé de cette interrogation identitaire propre à l'être humain. En effet, si le locuteur s'interroge sur son identité tout en se réduisant à un animal ou à un geste, l'existence même de la question identitaire est remise en cause. De même, les interrogations alternatives comme «¿eres del hombre o de la nada? ${ }^{16}$, proposent un éventail de réponses possibles qui restent pourtant inexistantes dans les textes. Le locuteur pousse même le vice jusqu'à enchaîner de multiples questions, selon ce qu'on appelle pour l'entretien psychiatrique les questions 《mitraillette ${ }^{17}:$ « ¿Y cómo podría hacerlo? ¿Quién era el Hombre Amarillo? ¿Cuándo había llegado a la ciudad? " ${ }^{18}$, "Quién es el ciervo / y qué la página / y dónde la rosa / húmeda del poema / es la interrogación que siniestra me formulo / cuando cae la página » ${ }^{19}$, ou encore "¿Qué dice la flor? ¿Qué dice la simiente? / ¿Qué dice cuando cae la frente? / ¿Qué Dios infecundo llora en el poema? $»^{20}$. Néanmoins, cette profusion interrogative de rythmes ternaires semble avoir l'effet inverse de celui escompté puisque la réponse n'est toujours pas effective.

Quant aux questions indirectes des recueils panériens, elles présentent systématiquement une négation les annulant et mettant en échec toute réponse possible : «No sé quién soy, ni quién los militares ${ }^{21}$, «sin saber en qué el alma consiste ${ }^{22}$. Le fait de nier le statut de question se retrouve également dans la manipulation de la ponctuation par

\footnotetext{
9 PANERO, Leopoldo María, Poesía completa (2000-2010), Madrid, Visor Libros, 2012, p. 166.

${ }^{10}$ PANERO, Leopoldo María, Poesía Completa (1970-2000), op. cit., p. 277.

11 Ibid, p. 37.

12 PANERO, Leopoldo María, Poesía completa (2000-2010), op. cit., p. 481.

${ }^{13}$ Ibid, p. 407.

${ }^{14}$ Ibid, p. 431.

${ }^{15}$ Ibid, p. 147.

16 PANERO, Leopoldo María, Poesía Completa (1970-2000), op. cit., p. 384.

${ }_{17}$ SHEA, Shawn Christopher, La conduite de l'entretien psychiatrique : l'art de la compréhension, Paris, Elsevier, 2005, p. 44.

18 PANERO, Leopoldo María, Poesía Completa (1970-2000), op. cit., p. 48.

${ }^{19}$ PANERO, Leopoldo María, Poesía completa (2000-2010), op. cit., p. 45.

${ }^{20}$ Ibid, p. 388.

${ }^{21}$ PANERO, Leopoldo María, Poesía Completa (1970-2000), op. cit., p. 381.

22 PANERO, Leopoldo María, Poesía completa (2000-2010), op. cit., p. 369.
} 
Panero qui met à mal l'existence même de l'interrogation en supprimant totalement les points d'interrogations, marque double et pourtant distinctive de cet acte de parole: "Quién soy debajo de mí, quién fuiste tú », ou en supprimant le point d’interrogation d'ouverture de la question, ce qui nous laisse croire jusqu'à la fin de la phrase à une prosodie déclarative : "será la muerte como un vino? / habrá mujeres en la tumba? » ${ }^{24}$ À l'inverse, nous trouvons des occurrences interrogatives sans point d'interrogation final, ce qui rend forcément impossible une quelconque réponse puisqu'elles restent en suspens, comme prolongées à l’infini : «¿Qué mano de madre, qué oración susurran / luna tras luna los labios de la luna $»^{25}$. La légitimité de la question semble de plus en plus mise en doute puisque cette interrogation est certes proférée, mais le locuteur lui-même ne la trouve pas adéquate. Ce n'est même plus la réponse qui est inexistante, mais la question elle-même qui n'arrive pas à se formuler correctement : «¿Por qué no bailar, ahora, el Danubio Azul? No es ésa, claro, la pregunta, pero mientras tanto, mientras me atrevo y no me atrevo ${ }^{26}$, « ¿estás triste en el cielo? / ¿Qué sentido tiene decir eso? ${ }^{27}$. Face à l'absence généralisée de réponses, se pose donc également la question du mauvais choix et de la mauvaise formulation des interrogations. Ne serait-ce pas une forme de rejet, consciente ou inconsciente, de l'interrogation et de l'interrogatoire-diagnostic comme socle de l'entretien psychiatrique, tel qu'il est théorisé notamment par Othmer et Othmer, ou Shawn Christopher Shea depuis la fin des années 80 au sein de l'institution psychiatrique que connaît si bien Leopoldo María Panero ? En effet, il pourrait s'agir ici de la négation du principe constitutif de l'interactivité psychiatrique définie sous la forme: "L'un des participants se désigne comme celui qui conduit l'entrevue et vise des objectifs précis tandis que l'autre participant assume en général le rôle de celui qui répond aux questions $»^{28}$. Cette fuite au-delà de l'échange question-réponse détruirait ainsi la soi-disant efficacité du clinicien : «C'est la qualité de nos questions, et non la quantité de nos propos, qui convainc [le patient] de l'aide offerte par les connaissances du clinicien $»^{29}$. Ce rejet de la méthode psychiatrique, cheval de bataille de Panero comme le signale par ailleurs Túa Blesa ${ }^{30}$, a lieu à travers la destruction du couple Question/Réponse dans ses poèmes. Ne serait-ce pas là une évacuation totale de la question du fait de son contenu anxiogène : «il y a dans la question, par-delà le non savoir qu'elle explicite, une part d'incompréhensible, de crise, de peur, d'angoisse $»^{31}$. Si même des questions extrêmement ouvertes et laissant la place à un champ de réponses infini, telles que "¿Qué es la verdad? »" sont confrontées à la nonréponse, la voix poétique va très vite se rediriger vers des interrogations assumant cette fois l'absence de réponse effective. Il s'agit bien sûr de la question rhétorique qui « ne sert plus à rien d'autre qu'à marquer une incertitude, et [elle] a perdu sa valeur d'interrogation, son pouvoir d'obliger autrui à répondre $»^{33}$. Le caractère rhétorique d'une question est indiqué par sa négation externe, « le locuteur n’interroge que fictivement pour suggérer qu'il refuse

\footnotetext{
23 PANERO, Leopoldo María, Poesía Completa (1970-2000), op. cit., p. 398.

${ }^{24}$ Ibid, p. 262.

${ }^{25}$ Ibid, p. 211.

${ }^{26}$ Ibid, p. 53.

${ }^{27}$ Ibid, p. 428.

${ }^{28}$ SHEA, Shawn Christopher, La conduite de l'entretien..., op. cit., p. 6.

${ }^{29}$ Ibid, p. 34.

30 « el poeta, quien, mientras sale y entra en manicomios, da a conocer el "Manifiesto del (II) Colectivo de Psiquiatrizados en lucha" en El viejo topo en 1980 », BLESA, Túa, Leopoldo María Panero, el último poeta, Madrid, El Club Diógenes, 1995, p. 18.

${ }^{31}$ GELAS, Nadine, «La question dans les romans de Marguerite Duras », in La Question, op. cit., p. 364.

32 PANERO, Leopoldo María, Poesía completa (2000-2010), op. cit., p. 310.

${ }^{3}$ DUCROT, Oswald, Dire ou ne pas dire, principes de sémantique linguistique, Paris, Hermann, 1972, p. 17.
} 
vigoureusement l'idée exprimée $»^{34}$, et c'est ce que nous pouvons repérer dans les occurrences suivantes: "¿Acaso no amas / que yo te orine?" " " ¿no era aquello una victoria? " " " ¿no será Olana que me observa / quizá, tal vez, desde una nube / en forma de Espía? ${ }^{37}$. Par la subversion de l'ordre linguistique, le poète impose le néant comme seule réponse possible à ses questions existentielles puisque « la pregunta retórica que se hace el poeta tiene una respuesta sabida por poeta y lector: nadie le responderá $\rangle^{38}$.

Par conséquent, la question, qui motive et structure toute tentative de recherche identitaire, est ici totalement détruite par Panero par sa transgression de l'obligation d'une réponse qui est de facto avortée. La prépondérance des questions rhétoriques mérite que nous accordions notre intérêt à la portée de cet acte interrogatif par définition en échec : il est répertorié dans la Gramática descriptiva de la lengua española au sein de la catégorie des exclamations et, comme le signale la linguiste Nathalie Fourier, "l'interrogation dite rhétorique $[\ldots]$ est proche de l'exclamation ${ }^{39}$. Ainsi, nous allons désormais analyser ce rapprochement entre interrogation et exclamation, et essayer de voir pourquoi la voix poématique glisse de plus en plus vers le choix de l'exclamation pour sa quête identitaire.

\section{Évitement de l'interrogation et évidence (salutaire ?) de l'exclamation}

Ce glissement progressif vers une exclamation, qui finit par supplanter totalement l'interrogation à partir du recueil de 2000, Teoría del miedo, et jusqu'à Sombra de 2008, se produit de manière naturelle, du fait des caractéristiques linguistiques très proches de ces deux actes illocutoires. Cette contiguïté est notamment due à leur schéma intonatif focalisé sur un terme précis, à leur capacité d'exprimer l'état du locuteur, mais également à leur morphologie constitutive puisque les mêmes morphèmes expriment l'interrogation et l'exclamation dans de nombreuses langues. Il existe d'ailleurs des cas ambigus et intermédiaires, "susceptible[s] d'être interprété[s] interrogativement ou exclamativement » ${ }^{40}$ que Navarro Tomás appelle des «preguntas exclamativas » ${ }^{41}$ ou Ozzello des «questclamations » ${ }^{42}$, et c'est également ce que précise le linguiste Alonso Cortés :

No es extraño que haya casos intermedios entre exclamativas e interrogativas pronominales, y que una pregunta pueda ser simultáneamente exclamativa, y una exclamativa adquiera tonos interrogativos. ${ }^{43}$

\footnotetext{
${ }^{34}$ FOURNIER, Nathalie, L'aparté dans le théatre français du XVII au XXe siècle. Étude linguistique et dramaturgique, Leuven, Peeters Leuven, 1991, p. 173.

35 PANERO, Leopoldo María, Poesía Completa (1970-2000), op. cit., p. 164.

${ }^{36}$ Ibid, p. 116.

${ }^{37}$ Ibid, p. 215.

38 ALONSO CORTÉS, Ángel, Exclamación en español, estudio sintáctico y pragmático, Madrid, Minerva Ediciones, 1999, p. 14-15.

${ }^{39}$ FOURNIER, Nathalie, L'aparté dans..., op. cit., p. 166.

${ }^{40}$ MARTIN, Robert, Langage et croyance : les univers de croyance dans la théorie sémantique, Bruxelles, Mardaga, 1987, p. 95.

${ }^{41}$ ALONSO CORTÉS, Ángel, "Las construcciones exclamativas. La interjección y las expresiones vocativas", in Gramática descriptiva de la lengua española (vol 3), Madrid, Espasa, 1999, p. 4004.

42 DUBOIS, Caroline, La grammaire de l'exclamation: aspects théoriques, français de référence et français québécois, Sherbrooke, Université de Sherbrooke, 2000, p. 52.

43 ALONSO CORTÉS, Ángel, "Las construcciones exclamativas...”, op. cit., p. 4004.
} 
C'est un cas hybride que Panero utilise, et l'hésitation entre interrogation et exclamation est ici renforcée par l'absence totale de ponctuation qui ne nous donne donc aucun indice sur la nature même de cet acte illocutoire: «Cómo duele en la sombra desear cuerpos muertos. $»^{44}$, «Y para qué la lluvia / y el papel siempre en vano ${ }^{45}$, ou «quién pudiera quebrar estos barrotes como espigas ${ }^{46}$. Cet emploi de questions exclamatives nous signale d'ores et déjà la propension panérienne à tendre vers l'exclamation.

Nous pourrions nous demander à présent en quoi les exclamations seraient plus favorables à la recherche identitaire panérienne, face à des interrogations incapables de résoudre quelque question existentielle que ce soit, comme nous l'avons vu précédemment. Cette préférence tiendrait au sémantisme intrinsèque de l'exclamation qui s'appuie sur l'évidence, sur la vérité exprimée avec force et sur la «condición de sinceridad »" Puisque la voix poématique panérienne est en proie au doute généralisé, quoi de plus efficace que de préférer un acte illocutoire expressif spontané et dont l'intensité psychologique, syntaxique et prosodique de haut degré peut appuyer la capacité d'auto-conviction de la voix poétique. Il s'agit, d'après la linguiste Caroline Dubois, non pas de simples assertions, mais $\mathrm{d}^{\prime}$ «hyperassertions $»^{48}$, puisque le locuteur n'apporte pas de nouvel élément, mais exprime ce qui est déjà pour lui une certitude : «La phrase exclamative -toute phrase exclamativeprend appui sur l'évidence : $p$ est asserté avec force ${ }^{49}$, et c'est ce que souligne également Nathalie Fournier : «l'exclamation produit un effet d'assertion superlative et incontestable, et elle a pour fonction d'authentifier et de légitimer l'énonciation $»^{50}$. Le locuteur panérien, en employant fréquemment les exclamations, puis en les préférant majoritairement dans les années 2000, se retrouve donc pour la première fois dans une sensation d'évidence et au premier plan de la subjectivité du langage. Cela laisserait ainsi plus de place à une définition identitaire profonde, à la libération des refoulements, à l'éclosion des interdits car les exclamations ne s'intègreraient plus dans une démarche d'interrogatoire, mais bien dans une perspective d'exutoire. Le locuteur, en exprimant par l'exclamation ses réactions au lecteur, à la page, à lui-même (et non pas en les décrivant ou les racontant), se situe alors dans une sorte de performativité de l'émotion mise en valeur par le rôle actif du sujet analysant prôné par Lacan (et non plus un sujet analysé ou interrogé). Ces exclamations apparaissent donc chez Panero de manière prépondérante et comme tentative de solution face au manque de définition identitaire. Nous constatons que l'évidence qui s'impose à la voix panérienne est celle de l'ennui «Qué largo es el día / sin término las noches / sin término los días $~^{51}$, celle de l'échec "iQué error ser yo, debajo de la luna! " " " Cuánto oro hay en la ruina $»^{53}$, «Viva el perder, viva el brillo oscuro de la ruina $~^{54}$, «Cuán perfecta es la silueta del desastre $»^{55}$, celle du vide et de la solitude « qué oscura es la luz, luz de la nada $»^{56}$, «Dios mío qué solo se queda el poema $»^{57}$, ou de la mort « ¡Villon ha muerto ahorcado! ${ }^{58}$,

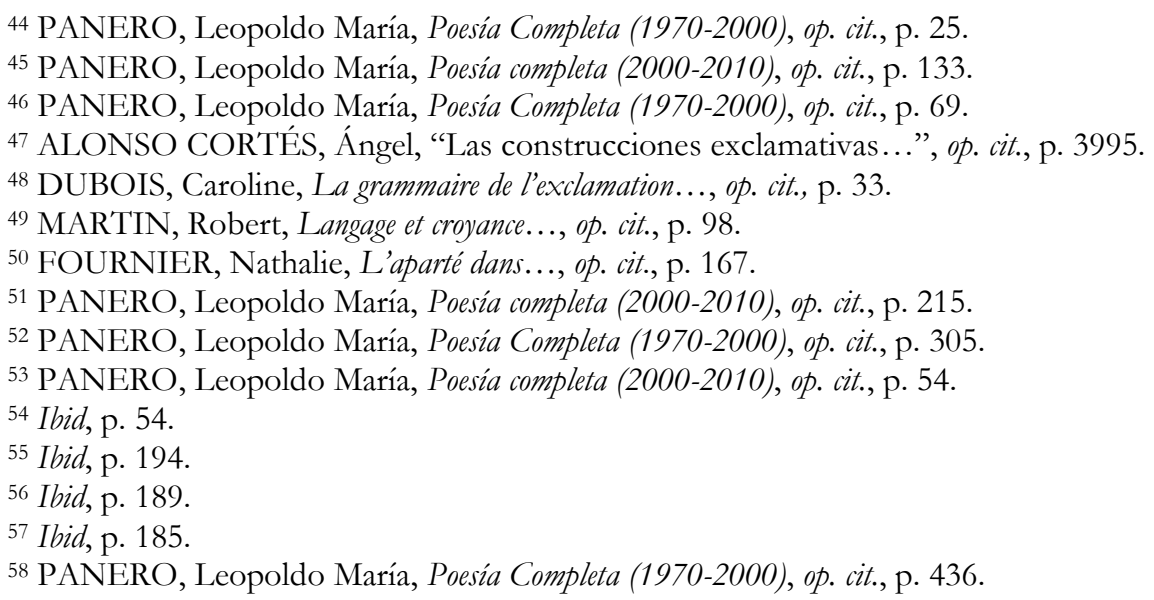


" ¡también tú morirás en mí! » ${ }^{59}$. Ces concepts extrêmement obscurs sont en outre qualifiés par des adjectifs au pessimisme sombre «horrenda $»^{60}$, «atroz $»^{61}$, ou paradoxalement, à l'exaltation hyperbolique «perfecta $»^{62}$.

Néanmoins, il convient de nuancer nos propos quant à la charge d'évidence exprimée par les exclamations panériennes et à la solution identitaire proposée ici : en effet, le linguiste Robert Martin nous dévoile la facette négative cachée dans le sémantisme de l'exclamation :

Pour être évidente, l'assertion exclamative n'en crée pas moins une tension contradictoire. « Il est là ! » Je le vois là devant moi. Mais qui l'eût cru ? Tout donnait à penser qu'il ne serait pas là. Ainsi, la phrase exclamative évoque dans quelque image d'univers des mondes où contradictoirement $p$ est faux. ${ }^{63}$

Ces exclamations présupposent un mensonge qui détruit par conséquent les fondements identitaires que la voix poématique pouvait penser y trouver. De plus, la marque typographique donnant entre autres choses son statut d'exclamation est très majoritairement effacée comme c'était le cas pour les points d'interrogation : il s'agirait ici d'une volonté de manipulation de l'intonation afin de retirer à ces occurrences leur caractère exclamatif ? De la sorte, cette voix panérienne instaure un silence définitif, surtout dans les derniers recueils, silence dont on ne semble pouvoir s'échapper, et tout aussi signifiant que l'est le cri également associé à l'exclamation chez Panero : " "iLeonela no existe!" grité, pero no quisieron escucharme »", "gritó : “Qué vergüenza estar vivo, y no ser querido" $»^{65}$, « nadie lo sabe: aúlla $»^{66}$. Pour terminer notre analyse de la déconstruction des fondements linguistiques de la quête du moi panérien, nous nous interrogerons donc sur l'apparition conjointe du cri et du silence chez le poète.

\section{Cri et silence, « ah » et « oh » : utopie de la recherche identitaire}

Túa Blesa consacre quelques pages à l'emploi des interjections exclamatives «ah » et « oh» à propos des recueils panériens de 2002, Buena nueva del desastre et Los señores del alma, mais il convient tout de même de préciser que ces occurrences exclamatives apparaissaient dès Asi se fundó Carnaby Street en 1970. Elles étaient certes beaucoup moins envahissantes que dans les recueils publiés depuis 2002 jusqu'à aujourd'hui, où l'on trouve une moyenne d'une interjection et demie par poème selon le critique aragonais, jusqu'à atteindre parfois cent occurrences dans un même recueil ${ }^{67}$. Ces interjections, de par leur nature formelle, n'apportent aucune information conceptuelle, et dans le cas précis de «ah » et «oh», elles ne se caractérisent pas non plus par un sémantisme particulier: "algunas interjecciones,

\footnotetext{
${ }^{59}$ Ibid, p. 438.

${ }^{60}$ PANERO, Leopoldo María, Poesía completa (2000-2010), op. cit., p. 258.

${ }^{61}$ Ibid, p. 113,116 et 269.

${ }^{62}$ Ibid, p. 190 et 194.

${ }^{63}$ MARTIN, Robert, Langage et croyance..., op. cit., p. 99.

${ }^{64}$ PANERO, Leopoldo María, Poesía Completa (1970-2000), op. cit., p. 42.

65 PANERO, Leopoldo María, Poesía completa (2000-2010), op. cit., p. 504.

66 PANERO, Leopoldo María, Poesía Completa (1970-2000), op. cit., p. 306.

${ }^{67}$ Nous pourrions nous demander si cette invasion textuelle des « ah» et «oh» ne pourrait pas avoir un lien quelconque avec l'arrivée de Leopoldo María Panero aux îles Canaries, à cette époque-là.
} 
como «ah» $[. .$.$] , no se pueden relacionar biunívocamente con una actitud emocional { }^{68}$. Non seulement il est difficile de définir leurs sens, mais en outre, Panero les utilise sans coïncider avec les types d'occurrences pragmatiques que relèvent les spécialistes :

La interjección "ah" sirve para marcar la toma de turno de palabra y, cuando repara enunciados anteriores del mismo hablante, puede marcar el mantenimiento del turno. Además de la función reparadora, también se emplea para manifestar la recepción de información, aunque no sea necesario que manifieste la aprobación. También se emplea para solicitar información adicional, sea en clarificaciones o en reparaciones. ${ }^{69}$

En effet, chez Panero, les interjections exclamatives «ah » et «oh» ne servent qu'à insister sur le terme focalisé ou à introduire une apostrophe litanique, du fait de leur fonction vocative, et elles ne transmettent pas une émotion précise ni définitive comme le souligne Túa Blesa : "Por tanto, momentos discursivos que son de énfasis o apelación, pero no de designación. Así “ah", "oh" son palabras que se resisten a que se les atribuya la condición de signos $»^{70}$. Cette "carencia de significado $»^{71}$ des interjections relevée par Blesa se retrouve notamment dans les vers suivants : " jah los hermanos, los hermanos invisibles que/ florecen/ en el Terror! » ${ }^{72}$, «Oh mi cabeza, mi cabeza, mi pobre cabeza! » ${ }^{73}$, « joh! la mano del hombre que tiembla / ante una inmensa escritura $»^{74}$. Il s'agit en réalité de mots creux qui peuvent former une phrase à eux tout seuls, ou introduire tout simplement une exclamation: c'est donc un discours de plainte in absentia qui envahit totalement l'espace textuel (notamment avec des exclamations que nous pourrions qualifier de «mitraillette» sur le modèle des interrogations et des rythmes ternaires : " ¡Oh! pecado en la sien, ¡Oh! flor de la locura / ¡Oh! pecado del verso $\rangle^{75}$ ). Ainsi, cela serait un langage qui vient détruire l'espoir d'une exclamation salvatrice au sein de la recherche identitaire. De plus, «ah » et «oh» peuvent sortir du langage articulé pour devenir de simples cris : «están pues en la lengua, pero exceden ese espacio - o dicho de otro modo, aún no llegan a él - y se aproximan a lo que se denomina grito, o quizá lo son sin más. $»^{76}$.

Le cri dérive de l'incapacité de se faire comprendre, comme le signale fort justement Elias Canetti :

Hablamos con alguien, pero de forma que no nos entienda. Seguimos hablando, y el otro entiende aún menos. Gritamos, él nos devuelve el grito, y la exclamación que en el ámbito de la gramática lleva una vida miserable, se apodera del lenguaje. ${ }^{77}$

Cri et exclamation sont donc bien liés et justement, le linguiste John Eliot Gardiner nous indique que les interjections exclamatives sont plus des $«$ cris signifiants ${ }^{78}$ que des mots.

\footnotetext{
${ }^{68}$ FÁBREGAS, Antonio, GIL, Irene, "Algunos problemas de la interjección en lexicografía", in 25 años de lingüistica aplicada en España: Hitos y Retos, AESLA, http://www.um.es/lacell/aesla/contenido/indice-area5.html, p. 635.

${ }^{69}$ Ibid, p. 637.

${ }^{70}$ BLESA, Túa, " “Ah”, “oh”: la escritura (última) de Leopoldo María Panero », in Tránsitos: escritos sobre poesía, Valencia, Tirant lo Blanch, 2004, p. 138.

${ }^{71} \mathrm{Ibid}$.

72 PANERO, Leopoldo María, Poesía Completa (1970-2000), op. cit., p. 151.

${ }^{73}$ Ibid, p. 375.

${ }^{74}$ Ibid, p. 419.

75 PANERO, Leopoldo María, Poesía completa (2000-2010), op. cit., p. 291.

${ }^{76}$ BLESA, Túa, "“Ah”, "oh”: la escritura (última) de Leopoldo María Panero... », op. cit., p. 138.

${ }^{77}$ CANETTI, Elias, La conciencia de las palabras, México, FCE, 1981, p. 58.
} 
Ces monosyllabes «ah» et "oh», toniques et marquant un accent syncopé au début des vers panériens ${ }^{79}$, nous montrent à quel point la recherche identitaire de la voix poématique a échoué puisqu'ils sont "un grito de inseguridad " lancé de manière paroxystique et transgressive par ceux qui ne se sentent pas entendus. Leopoldo María Panero va encore plus loin car ces interjections «ah » et «oh», ces syllabes réitérées, deviennent le thème central, le seul message métapoétique transmis par le poème qui se retrouve donc vide de sens : «Ah, el ciervo azul de la página /de la página que dice $\mathrm{Ah} / \mathrm{y} \mathrm{Oh}$ » $^{81}$ « Dios mío qué solo se queda el poema / Que dice oh y ah, como un ángel para la tumba » ${ }^{82}$, ¡ $\mathrm{Oh}$ ! tú sílaba del papel, única mano / para destripar sobre la página $»^{83}$. Ce cri extériorisé est ce qui est de plus intime à la voix poétique et d'ailleurs, n'exprimerait-il pas une qualité prélinguistique de l'interjection, presque un retour au cri premier de l'enfant ? Mikkel Borch-Jacobsen, à propos de Lacan, nous signale également que ce «cri humain est d'entrée de jeu une demande de reconnaissance ${ }^{84}$, et Lacan lui-même dans un de ses séminaires s'interroge : «Qu'est-ce que c'est que ce cri ? Qui l'entendrait, ce cri que nous n'entendons pas ? Sinon justement qu'il impose ce règne du silence ${ }^{85}$. Nous pouvons tenter d'avancer qu'il s'agit là sûrement de la meilleure forme de catharsis possible pour la voix panérienne qui accepterait et apprivoiserait enfin le silence, l'ineffable du langage et l'impossibilité de résoudre sa problématique identitaire.

Les stratégies discursives que la voix panérienne met en place sont donc toutes vouées à l'échec, et cette quête absolument utopique du moi poétique apparaît dans les vers par la présence simultanée d'occurrences évoquant le cri et sa syllabe correspondante «ah » ou « oh », le silence, le néant et la destruction, comme le fait remarquer Túa Blesa :

"ah" y "oh" son, sí, momentos del decir, pero de un decir donde la única posibilidad es ya la de decir nada [...] Escritura, por tanto, la de Panero que sería el trazo en el vacío, el trazo vacío, el vacío del trazo, una escritura de los signos de la nada. ${ }^{86}$

C'est le cas dans les exemples suivants qui mêlent tous ces champs lexicaux caractéristiques des obsessions panériennes : "lucha de las palabras con el viento / lucha de las sílabas y el viento / de la sílaba que grita a la palabra "nada" $»^{87}$, " oh tiniebla del llanto / infierno del poema / canto atroz al silencio / blanco de la sílaba / impura » ${ }^{88}$, « oh esplendor de la nada / en donde caen las sílabas / una tras otra, siguiendo / el camino perfecto de la nada ${ }^{89}$, ou encore «Oh amor impuro! Amor de las sílabas y de las letras / que destruyen el mundo »". La destruction via la syllabe «ah» ou «oh» est bien revendiquée chez Leopoldo María Panero et semble même faire l'objet d'une véritable déclaration d'amour, d'une foi

\footnotetext{
${ }^{78}$ MOESCHLER, Jacques, REBOUL, Anne, Dictionnaire encyclopédique de pragmatique, Paris, Seuil, 1994, p. 54.

79 À ce sujet, Ramiro Oviedo suggérait fort justement que ces syllabes-interjections seraient une forme de coup de poing et de trou créés par la transe monotone du cri, qui permettraient à la langue de s'engouffrer à l'intérieur du vers, comme attirée par un aimant.

80 ALONSO CORTÉS, Ángel, Exclamación en español, op. cit., p. 15.

${ }^{81}$ PANERO, Leopoldo María, Poesía completa (2000-2010), op. cit., p. 118.

82 Ibid, p. 185.

${ }^{83} \mathrm{Ibid}$, p. 305.

${ }^{84}$ BORCH-JACOBSEN, Mikkel, Lacan, le maître absolu, Paris, Flammarion, 1990, p. 245.

${ }^{85}$ LACAN, Jacques, Séminaire XII, Problèmes cruciaux de la psychanalyse (non publié), leçon du 17/03/1965, http://www.ecole-lacanienne.net/stenos/seminaireXII/1965.03.17.pdf

86 BLESA, Túa, “"Ah”, “oh”: la escritura (última) de Leopoldo María Panero... », op. cit., p. 139.

${ }^{87}$ PANERO, Leopoldo María, Poesía completa (2000-2010), op. cit., p. 76.

${ }^{88}$ Ibid, p. 113.

89 Ibid, p. 156.

${ }^{90}$ PANERO, Leopoldo María, Poesía Completa (1970-2000), op. cit., p. 433.
} 
paradoxale vouée à l'anéantissement du langage : «Oh destrucción mi Beatriz segura »" «Oh TÚ destrucción, mi único amor »". Cette articulation dialectique du cri et du silence au sein d'une destruction violente de la langue nous fait revenir au statut initial de doute, d'absence de réponses que nous analysions dans notre première partie. Cela est d'autant plus vrai que, dans les tout derniers recueils panériens à partir de 2008 et Sombra, les interrogations non-saturées font leur retour après leur évitement momentané pendant quelques années, et comme l'affirme Christian Plantin : «si les questions sont insaturées, c'est que la vraie réponse n'existe pas ${ }^{93}$. En réalité, c'est comme si cette "escritura de la transgresión ${ }^{94}$ assumait finalement son impuissance, la destruction de tous ses codes, voire son autodestruction, puisque la logophagie ${ }^{95}$ théorisée par Túa Blesa est justement présentée comme une forme de renoncement, comme une «realización sistemática de un programa de destrucción ${ }^{96}$. Il ne faudrait pas néanmoins sous-estimer la portée de cet acte de silence qui, bien qu'étant un échec au sein de la recherche identitaire, a une charge d'indicible très importante, comme le rappelle Pierre Van den Heuvel : " on sait bien que le silence "parle", que son "éloquence" joue un rôle capital dans la communication et qu'il peut être aussi effrayant que le cri »", "l'écriture apparait comme une activité vaine, mais nécessaire malgré tout, qui sert plus à exorciser qu'à communiquer, plus à immoler la langue qu'à la renouveler $\nu^{98}$. Il ne s'agirait donc plus du cri ni du mutisme de l'aliéné, mais bien du refus de s'enfermer dans une définition fixe et de l'acquisition d'une vision lucide quant à l'impossibilité de résoudre la moindre problématique identitaire par le biais de l'écriture.

Pour conclure à propos de cet échec de l'utopie de la recherche identitaire panérienne, il nous faut rappeler que nous nous sommes d'abord penchés sur l'acte interrogatif en tant que clé de voûte de la quête du moi poétique. Suite à de multiples transgressions de cet acte de langage, il nous est apparu comme non-saturé, remis en question et rejeté catégoriquement au profit d'une exclamation censée exprimer l'évidence qui manquait justement au locuteur panérien, dès qu'il s'agissait de chanter sa personne ou le monde qui l'entoure. Or, l'exclamation est elle aussi détruite de par son contenu sémantique d'imaginaire et de mensonge, et par la prépondérance et l'envahissement exponentiel des interjections «ah» et «oh» qui détournent la voix poématique de sa recherche identitaire initiale, en préférant le cri et le silence, en somme, l'aveu d'une quête identitaire vaine, mais dont la destruction de l'utopie est porteuse d'une créativité profondément excessive et subversive.

\footnotetext{
91 PANERO, Leopoldo María, Poesía completa (2000-2010), op. cit., p. 56.

92 Ibid, p. 433.

93 PLANTIN, Christian, "Questions $\rightarrow$ argumentations $\rightarrow$ réponses », in La Question, op. cit., p. 69.

${ }^{94}$ BLESA, Túa, “"Ah”, “oh”: la escritura (última) de Leopoldo María Panero... », op. cit., p. 127.

${ }^{95} \mathrm{Cf}$ son ouvrage intitulé Logofagias, los trazos del silencio, publié à l’Université de Saragosse en 1998.

96 BLESA, Túa, «La destruction fut ma Béatrice, I », in Poesía Completa (1970-2000), op. cit., p. 9.

${ }^{77}$ VAN DEN HEUVEL, Pierre, Parole, mot, silence : pour une poétique de l'énonciation, Paris, Corti, 1985, p. 67.

98 Ibid, p. 84.
} 